\title{
DIE REPRÄSENTATION DER KAISERLICHEN MACHT IN DEN INSCHRIFTEN ROMS UND DES IMPERIUM ROMANUM Von GÉZA ALFÖLDY
}

\author{
Silvio Panciera \\ septuagenario \\ amico optimo et \\ praestantissimo
}

Vor kurzem schrieb Silvio Panciera: „In passato si è molto insistito, contrapponendola alla "soggettività" della narrazione storica, sulla presunta "oggettività" della comunicazione epigrafica. Non così negli ultimi anni, in cui, se mai, si è andato sempre più evidenziando quanta parte abbia la volontà di autorappresentazione (soggettiva quant'altra mai) nella scelta di realizzare un'iscrizione in un certo modo, su un certo monumento ed in un certo luogo." Diese Art der epigraphischen Kultur Roms, die sie etwa von der archivarischen Dokumentation von Daten in den altorientalischen Keilschriftenkulturen oder in der kretisch-mykenischen Welt unterscheidet, war bereits während der Republik unverkennbar. Nach eher bescheidenen Anfängen entfaltete sich aber diese epigraphische Kultur in großem Stil erst seit Augustus, der die Bedeutung des Mediums „Inschrift“ - oder besser gesagt: die publizistische Wirksamkeit von Monumenten, zu denen auch eine Inschrift gehörte - viel besser als seine Vorgänger erkannte. Erst unter Augustus - damals aber explosionsartig - begann in Rom und auch in den Provinzen die Ausbreitung einer epigraphischen Kultur in einem noch nie dagewesenen Umfang. Sie zielte darauf, daß die Wertvorstellungen und das Selbstverständnis der Herrschenden, aber auch der sich daran orientierenden breiteren Bevölkerungsmassen durch einprägsame Botschaften zum Ausdruck gebracht und zugleich auf Dauer bewahrt wurden. ${ }^{2}$ Im Mittelpunkt

\footnotetext{
${ }^{1}$ S. Panciera, in: G. Alföldy - S. Panciera (Eds.), Inschriftliche Denkmäler als Medien der Selbstdarstellung in der römischen Welt (Heidelberger Althistorische Beiträge und Epigraphische Studien 36), Stuttgart 2001, 8. - Armin U. Stylow danke ich für bibliographische Hinweise.

2 Uber die explosionsartige Entwicklung der epigraphischen Kultur Roms unter Augustus siehe G. Alföldy, Gymnasium 98, 1991, 289-324 = Scienze dell'Antichità. Storia, Archeologia, Antropologia. Dipartimento di Scienze Storiche, Archeologiche, Antropologiche dell'Antichità. Università degli di Studi di Roma "La Sapienza" 5, 1991 [1994], 573-600 (italienisch); der gleiche Beitrag in polnischer
} 
dieser epigraphischen Kultur stand, entsprechend dem soziopolitischen Gefuige des Imperium Romanum, die Verherrlichung der Kaiser mit ihrer Macht, die ihnen die Möglichkeit bieten sollte, ihre Tugenden zugunsten der res publica zu entfalten und deren Schicksale durch geeignete Maßnahmen zu lenken. ${ }^{3}$

Die zentrale Bedeutung, die in der Selbstdarstellung von Herrschern der Thematisierung ihrer Macht zukommt, ist schon in den Res Gestae Divi Augusti voll zu erkennen. ${ }^{4}$ Mag dieses Dokument als ausführlicher Tatenbericht noch so einzigartig sein, für die Botschaften, die durch Inschriften verkündet wurden, ist es doch vielfach kennzeichnend. Nach der Überschrift handelt es sich um die res gestae, durch die Augustus orbem

Sprache: August $\mathrm{i}$ inskripcije: tradycja u innowacje. Narodtiny epigrafiki imperialnej (Xenia Posnaniensia III), Poznan 1994. Über diese Entwicklung in Hispanien siehe F. Beltrán Lloris (Ed.), Roma y el nacimiento de la cultura epigráfica en Occidente. Actas del Coloquio Roma y las primeras culturas epigráficas del Occidente mediterráneo (siglos II a. E. - d. E.) (Zaragoza, 4 a 6 de noviembre de 1992), Zaragoza 1995, darin u. a. mit Beiträgen von M. Mayer, G. Alföldy, J. M. Abascal, A. U. Stylow u. a. Für die Entwicklung der epigraphischen Kultur Hispaniens siehe auch G. Alföldy, in: M. Almagro-Gorbea - J. M. Alvarez Martínez et al., Hispania. El legado de Roma, Zaragoza 1998, 289301 = in: dies., Hispania. El legado de Roma, Zaragoza 1999, 324-337; vgl. außerdem dens., in: L. Hernández Guerra - L. Sagredo San Eustaquio - J. M. ${ }^{a}$ Solana Sáinz (Eds.), Actas del I Congreso Internacional de Historia Antigua "La Península Ibérica hace 2000 años" Valladolid 23-25 de Noviembre 2000, Valladolid 2001 [2002], 61-74; A. U. Stylow, in: G. Urso (Ed.), Hispania terris omnibus felicior. Premesse ed esiti di un processo di integrazione. Atti del convegno internazionale, Cividale del Friuli, 27-29 settembre 2001, Pisa 2002, 163-181.

${ }^{3}$ Zur Repräsentation der kaiserlichen Macht durch die epigraphischen Denkmäler siehe etwa $\mathrm{M}$. Horster, Bauinschriften römischer Kaiser. Untersuchungen zu Inschriftenpraxis und Bautätigkeit in Städten des westlichen Imperium Romanum in der Zeit des Prinzipats (Historia Einzelschriften 157), Stuttgart 2001, vgl. dazu G. Alföldy, Journal of Roman Archaeology 15, 2002, 489-498 und Epigraphica 64, 2002, 113-145 (Bauinschriften); J. M. Højte, Roman Imperial Statue Bases. An Epigraphical Contribution to the Understanding of Roman Imperial Portrait Statues, unpubl. Manuskript Aarhus 2001 (Basen von Kaiserstatuen mit Inschriften von Augustus bis Commodus). Zur Rolle der verschiedenen Medien in der Selbstdarstellung der Herrscher siehe bes. A. Alföldi, Die monarchische Repräsentation im römischen Kaiserreiche, 3. Aufl. Darmstadt 1980 (Nachdruck der bahnbrechenden Studien des Autors aus den Jahren 1934 und 1935 aufgrund der Synoptik verschiedener Mittel der kaiserlichen Selbstdarstellung); P. Zanker, Augustus und die Macht der Bilder, München 1987 (Denkmäler der Kunst); M. Alföldi, Bild und Bildersprache der römischen Kaiser. Beispiele und Analysen (Kulturgeschichte der Antiken Welt 81), Mainz am Rhein 1999 (Münzbilder und weitere bildliche Darstellungen); vgl. noch W. Kuhoff, Felicior Augusto melior Traiano. Aspekte der Selbstdarstellung der römischen Kaiser während der Prinzipatszeit, Frankfurt am Main 1993 (Verwendung verschiedener Medien für einige Themen der Selbstdarstellung); für die Kunst der Spätantike unter diesem Aspekt siehe jetzt auch E. Mayr, Rom ist dort, wo der Kaiser ist. Untersuchungen zu den Staatsdenkmälern des dezentralisierten Reiches von Diocletian bis zu Theodosius II (Römisch-Germanisches Zentralmuseum Mainz, Monographien 53), Mainz 2002.

${ }^{4} \mathrm{Zu}$ den Res Gestae Divi Augusti siehe jetzt zusammenfassend D. Kienast, Augustus, Prinzeps und Monarch, 3. Aufl., Darmstadt 1999, 208 ff. mit Bibliographie. Als Edition wird hier H. Volkmann, Res Gestae Divi Augusti. Das Monumentum Ancyranum, 3. Aufl., Berlin 1969 benutzt. 
terrarum imperio populi Romani subiecit, ergänzt durch seine impensae zugunsten der Römer. Somit wird bereits am Anfang die einzigartige Machtstellung des Augustus verherrlicht, die zugleich Roms Macht verkörpert. Bekanntlich sagt zwar Augustus kurz vor dem Ende seines Berichts, daß er nach Abschluß der Bürgerkriege die res publica aus seiner potestas in diejenige von Senat und Volk übergab und daß seine potestas, im Gegensatz zu seiner auctoritas, mit der er alle Menschen übertraf, nicht größer war als die seiner Amtskollegen. ${ }^{5}$ Der Gesamteindruck des Lesers, der sich nicht so sehr für die subtilen Feinheiten konstitutioneller und ideologischer Kategorien, sondern für die Realitäten interessierte, dürfte jedoch ein anderer gewesen sein. Er konnte hier lesen, daß Augustus 13 Male Konsul und 37 Male Inhaber der tribunicia potestas war (die Konsulate des Augustus werden in den Res Gestae übrigens insgesamt gleich 17 Male, seine tribunizische Vollmacht funfmal erwähnt), ${ }^{6}$ daß er 21 Male als imperator gefeiert wurde, ${ }^{7} \mathrm{da} ß$ er auch in den Jahren, in denen er nicht Konsul war, mit konsularischem imperium handeln konnte. ${ }^{8}$ Dazu kamen die Hinweise auf seine vielen Siege und Triumphe, die „sein“ Heer, wenn nicht unter seiner persönlichen Führung, dann von seinen Legaten auf seinen Befehl und unter seinen Auspizien erzielte $;{ }^{9}$ darauf, daß in seinen Triumphzügen nicht weniger als neun gefangene Könige oder Kinder von Königen geführt wurden und daß neun andere Könige oder Königssöhne unter seinen Schutz flohen, ${ }^{10}$ auf die manibiae, aus denen er Prachtbauten finanzierte. ${ }^{11}$ Selbst seine impensae und die dadurch möglich gewordenen Leistungen stellten mit ihren Dimensionen zumindest wirtschaftliche Macht dar. ${ }^{12}$ Wo bei all dem die Grenze zwischen konstitutionell definierter potestas und persönlicher auctoritas verlief, dürfte den meisten Untertanen unklar und auch kaum von Interesse gewesen sein. In ihren Augen muß auch die auctoritas des pater patriae als Macht gegolten haben, der sie sich

\footnotetext{
${ }^{5}$ Res Gestae Divi Augusti 34. Zum augusteischen Begriff der auctoritas vgl. zusammenfassend L. Wickert, RE XXII 2, 1954, 2287-2290.

${ }^{6}$ Gesamtzahl der Konsulate und der Verleihungen der tribunicia potestas: Res Gestae Divi Augusti 4. Weitere Hinweise auf die Konsulate des Augustus: ebd. 1. 8 (bis). 15 (sexties). 16. 20 (bis). 21. 22. 34. 35; weitere Hinweise auf die tribunizische Vollmacht: ebd. 6. 10. 15 (bis).

${ }^{7}$ Ebd. 4, vgl. auch 21.

${ }^{8}$ Ebd. 8.

${ }^{9}$ Exercitus meus: ebd. 30; der Terminus ist dem kurz davor erwähnten exercitus populi Romani vergangener Zeiten deutlich gegenübergestellt. Vgl. auch den Terminus classis mea, ebd. 26. Feldzüge iussu und auspiciis des Augustus als Oberbefehlshabers: ebd. 4. 26. 30.

${ }^{10}$ Ebd. 4 und 32.

${ }^{11}$ Ebd. 15 und 21.

${ }^{12}$ Ebd. $15-23$ und add. 1.
} 
unterordnen mußten, von der sie abhängig waren, von deren Träger sie jedoch erwarteten, daß er sie beschützt und ihre Interessen vertritt.

Dem Leser der Res Gestae mußten aber sicher nicht nur die erwähnten und die übrigen Textinhalte als Indizien für eine noch nie dagewesene Macht erscheinen. Die Größe des Augustus wurde auch dadurch unterstrichen, daß dieser Text in einer bisher ungewohnten Weise auf zwei Erzpfeilern eingraviert war, die schon durch ihre Form und Größe beeindruckten. ${ }^{13}$ Und sie standen am Eingang des Mausoleums des Kaisers, des damals größten, u. a. mit zahlreichen Inschriften versehenen Grabmonumentes in Rom, ${ }^{14}$ in umittelbarer Nähe von zwei Obelisken, die selbst als Symbole der Herrschaft galten. Außerdem gab es von hier einen direkten Sichtkontakt zum solarium Augusti mit dem Montecitorio-Obelisken als Gnomon, das nicht nur die irdische Macht (u. a. durch den Hinweis auf die Unterwerfung Ägyptens durch Augustus in der Inschrift des Obelisken), sondern auch die kosmische Größe des Herrschers verkündete. ${ }^{15}$ Es sei hier angemerkt, daß die Ancyraner Kopie der Res Gestae an einer Seitenwand des Augustustempels zu lesen war, der sich dort an einer beherrschenden Stelle der Stadt erhebt; die Antiochenser Kopie wurde ebenfalls an einer prominenten Stelle der Stadt, im Bezirk des Herrscherkultes, zur Schau gestellt; die Kopie von Apollonia in Pisidien wurde innerhalb eines Temenos des Kaiserkultes auf einem großen Sockel eingemeißelt, auf dem die Statuen von Divus Augustus, Livia, Tiberius, Germanicus und Drusus minor standen. ${ }^{16}$

Die Verknüpfung von Text, äußerem Glanz der Inschrift, Inschriftenträger, architektonischem Rahmen und Einbindung in einen

\footnotetext{
${ }^{13}$ Ebd., Praef.

${ }^{14} \mathrm{Zu}$ diesem Monument siehe H. von Hesberg - S. Panciera, Das Mausoleum des Augustus. Der Bau und seine Inschriften (Bayerische Akademie der Wissenschaften, Philosophisch-historische Klasse, Abhandlungen N. F. 108), München 1994; die Inschriften des Mausoleums auch in CIL VI 4035640410.

${ }^{15}$ E. Buchner, Römische Mitteilungen 83, 1976, 319-365 und ebd. 87, 1980, 355-373; ders., Die Sonnenuhr des Augustus, Mainz am Rhein 1982; ders., in: E. M. Steinby (Ed.), Lexicon Topographicum urbis Romae III, Roma 1996, 35-37; Inschrift auf dem Sockel des MontecitorioObelisken: CIL VI 701-702 = ILS 91. Vgl. G. W. Bowersock, in: K. A. Raaflaub - M. Toher (Eds.), Between Republic and Empire. Interpretations of Augustus and His Principate, Berkeley - Los Angeles - Oxford 1990, 383-385.

${ }^{16}$ Volkmann, a.a.O. (Anm. 4) 4 f. mit älterer Literatur; H. Hänlein-Schäfer, Veneratio Augusti. Eine Studie zu den Tempeln des ersten römischen Kaisers (Archaeologica 39), Roma 1985, 185-196. 270. 289 f.; zur Ausstellung der drei Kopien der Res Gestae Divi Augusti in den genannten Städten siehe jetzt P. Botteri, in diesem Band, zu Antiochia unlängst auch St. Mitchell - M. Waelkens, Pisidian Antioch. The Site and its Monuments, London 1998, 113-173.
} 
breiteren räumlichen Kontext eignete sich bestens dazu, das Gefühl hervorzurufen, daß hier eine noch nie dagewesene Macht verherrlicht wurde. Diese Wirkung verstärkte sich dadurch, daß die Res Gestae zumindest in der Provinz Galatia, anscheinend dank der Initiative des dortigen Statthalters, ${ }^{17}$ durch Kopien auch einem Publikum außerhalb Roms und Italiens unmittelbar bekanntgemacht wurden. Mit all dem waren die Res Gestae ein durchaus repräsentatives Beispiel für die Lobpreisung von Herrschern durch Monumente mit entsprechenden Inschriften.

Über die inhaltlichen Elemente in den sog. „Kaiserinschriften“, durch die die Vormachtstellung der Herrscher hervorgehoben wird, genügen hier Stichworte. Einzigartig war schon ihre Nomenklatur, beginnend mit dem Namen des Begründers des Prinzipats Imperator Caesar Divi filius Augustus, einem noch nie dagewesenen Namen. ${ }^{18}$ Wie die nicht seltene Variante Caesar Augustus - nur ein nomen und cognomen, ohne das praenomen, eine damals unübliche Namenskombination - zeigt, entsprach die Nomenklatur des Ersten Princeps in den Augen seiner Zeitgenossen auch rein formell nicht einfach dem traditionellen System der tria nomina eines Bürgers, sondern war etwas Außergewöhnliches, ${ }^{19}$ wie erst recht die späteren Kaisernamen mit den langen Ahnenreihen und den Siegerbeinamen. Die Hinweise auf die Machtbefugnisse und Vorrechte der Kaiser in ihrer Titulatur, u. a. mit der Nennung der imperatorischen Akklamationen, der tribunizischen Gewalt und der Konsulate einschließlich der entsprechenden Iterationsziffern, seit Lucius Septimius Severus regelmäßig auch der prokonsularischen Vollmacht, brachten ihre Machtfülle zum Ausdruck. Zumindest ursprünglich müssen mit diesen Rangtiteln klare Vorstellungen über die kaiserliche Macht verbunden gewesen sein. So nannte sich Augustus im kürzlich gefundenen Edikt aus El Bierzo in Hispanien im Gegensatz zur gängigen Praxis auch proconsul, um zu betonen, daß er seine Maßnahmen, die er damals, von Rom abwesend, für peregrine Gemeinden in einer seiner Provinzen traf, auf diese Amtsgewalt stützte. ${ }^{20}$ Man konnte aber die Rangtitel ganz weglassen oder den Kaiser nur als pater patriae rühmen,

\footnotetext{
${ }^{17}$ W. Eck - A. Caballos - F. Fernández, Das senatus consultum de Cn. Pisone patre (Vestigia 48), München 1996, $285 \mathrm{f}$.

${ }^{18}$ Grundlegend R. Syme, Historia 7, 1968, 172-188 = ders., Roman Papers I, Oxford 1979, 361- 377, deutsch in: W. Schmitthenner, Augustus (Wege der Forschung CXXVIII), Darmstadt 1969, 264-290.

${ }^{19}$ G. Alföldy, Gymnasium 98, 1991, 316 f.

${ }^{20}$ Siehe dazu G. Alföldy, Zeitschrift fur Papyrologie und Epigraphik 131, 2000, 177-205, bes. $193 \mathrm{f} .=$ Minima Epigraphica et Papyrologica IV 2001, 6, 365-417, bes. 392-395 (revidierte Fassung in italienischer Sprache).
} 
wie dies beispielsweise schon die Einwohner der Baetica bei der Stiftung ihrer goldenen Statue auf dem Augustusforum taten; ${ }^{21}$ damit wurde ebenso die patria potestas wie auch die einzigartige auctoritas des Princeps angesprochen. Selbst die Nomenklatur des Monarchen konnte auf den einzigen Namen Augustus beschränkt werden, denn seine grenzlose Vormachtstellung war für die Untertanen schon dadurch hinreichend vergegenwärtigt. ${ }^{22}$ Auch spätere Kaiser wurden in den Inschriften, vor allem wenn sie in den epigraphischen Dokumenten ihrer Untertanen als deren Wohltäter verherrlicht wurden, innerhalb der zur Titulatur gefestigten Nomenklatur des Ersten Princeps Imp. Caesar ... Augustus oder Imp. ... Augustus oft mit einem einzigen Namen wie z. B. Traianus, Hadrianus, Antoninus usw. genannt. Wer in einer Inschrift einer derartigen Nomenklatur begegnete, dem war es klar, daß der so genannte Mann kein gewöhnlicher Mensch, sondern der Herr über alle Menschen war.

Noch deutlicher als die stereotypen Rangtitel mit Hinweis auf die tribunizische Vollmacht o. ä., deren Bedeutung später mehr und mehr verloren ging, dürften die Macht der Kaiser in den Augen der Untertanen ihre zunehmend gebrauchten inoffiziellen Beinamen und, wie auch in den Res Gestae, die konkreten Hinweise auf ihre Taten veranschaulicht haben. ${ }^{23}$ Die lange Reihe der epigraphisch bezeugten Beinamen, die seit Trajan erkennbar zunahmen, seit den Severern eine inflationäre Entwicklung aufwiesen und somit allmählich entwertet wurden, ist allgemein bekannt. Besonders unterstrichen sie u. a. die Sieghaftigkeit und damit die militärische Macht der Kaiser. Bezeichnend sind etwa die Steigerung und

\footnotetext{
${ }^{21}$ CIL VI 31267 cf. p. 3778. 4340 f. (mit weiterer Bibliographie) = ILS 103, siehe dazu ausführlich G. Alföldy, in: H.-J. Drexhage - J. Sünskes (Hrsg.), Migratio et Commutatio. Studien zur Alten Geschichte und deren Nachleben. Thomas Pekáry zum 60. Geburtstag, St. Katharinen 1989, 226-234 = ders., Studi sull'epigrafia augustea e tiberiana di Roma (Vetera 8), Roma 1992, 67-75, auch mit Hinweisen auf weitere Inschriften des Augustus, in denen aus seiner Rangtitulatur nur die Bezeichnung $p$ (ater) $p$ (atriae) erscheint. Auch spätere Herrscher wurden nicht selten in der gleichen Weise verherrlicht, unter ihnen besonders häufig Antoninus Pius.

${ }^{22}$ So z. B. in der Dedikationsinschrift für eine Statue des Augustus in Saguntum: CIL II $2 / 14,305$.

${ }^{23} \mathrm{Zu}$ den inoffiziellen Beinamen der römischen Kaiser siehe bes. A. Alföldi, a.a.O. (Anm. 3) 204-213; R. Frei-Stolba, Museum Helveticum 26, 1969, 18-39; reiche Materialsammlung auch unter Berücksichtigung anderer Quellen bei F. Taeger, Charisma, Studien zur Geschichte des antiken Herrscherkultes II, Stuttgart 1960, 226-473. Speziell für das 3. Jahrhundert siehe außerdem M. Peachin, Roman Imperial Titulature and Chronology, A. D. 235-284 (Studia Amstelodamensia ad epigraphicam, ius antiquum et papyrologicam pertinentia XXIX), Amsterdam 1990; für die Späte Kaiserzeit siehe auch A. Chastagnol, in: A. Donati (Ed.), La terza età dell'epigrafia. Colloquio AIEGL - Borghesi 86 (Bologna, ottobre 1986) (Epigrafia e Antichità 9), Faenza 1988, 12-38; Th. Grünewald, Constantinus Maximus Augustus. Herrscherpropaganda in der zeitgenössischen Überlieferung (Historia-Einzelschriften 64), Stuttgart 1990, u. a. mit den Listen 265-270 und 274-276.
} 
der Wandel der Siegerbeinamen invictus (seit Commodus und Septimius Severus), super omnes retro principes invictus oder invictissimus (seit Caracalla), victoriosus und victoriosissimus (seit Aurelian), victor statt invictus (regelmäßig seit 324, ohne daß der heidnisch klingende Beiname invictus bzw. sein Superlativ damals ganz aufgegeben worden wären), bis zu ubique vincens (zur Bezeichnung ausgerechnet des Arcadius und Honorius, unter denen ein großer Teil des Reiches verloren ging). ${ }^{24}$ Unbegrenzte Macht suggerierte z. B. die Lobpreisung der Herrscher als restitutor orbis (seit Severus Alexander). ${ }^{25}$ Die Hinweise auf die kriegerischen Taten der Kaiser folgten zunächst z. T. dem Vorbild der Res Gestae, z. B. die Inschrift auf dem wegen des britannischen Sieges errichteten Claudiusbogen in Rom, in der ähnlich wie in den Res Gestae der Kaiser als derjenige gelobt wird, der fremde Völker unter die Hoheit des römischen Volkes brachte und in der ebenfalls von besiegten Königen die Rede ist. ${ }^{26}$ Auch die Hinweise auf die Finanzierung großer Bauten aus den manubiae, so in der rekonstruierten Bauinschrift des Colosseum ${ }^{27}$ oder in der verlorenen Bauinschrift des Trajansforums, ${ }^{28}$ folgten dem augusteischen Vorbild. Die überdimensionale Macht der Herrscher wurde aber auch dann deutlich, wenn er z. B. als Bezwinger von Raum und Natur erschien. Augustus rühmte sich in den Inschriften der Meilensteine an der via Augusta, diese nach ihm benannte Straße Hispaniens usque ad Oceanum, bis zum Ende der Welt, d. h. bis Gades, ausgebaut zu haben. ${ }^{29}$ Erwähnt seien hier auch die Inschriften von

\footnotetext{
${ }^{24} \mathrm{Zu}$ den Formeln super omnes retro principes invictus 0 . ä. siehe A. Scheithauer, Zeitschrift für Papyrologie und Epigraphik 72, 1988, 155-177; zur Übernahme des Beinamens Victor statt Invictus durch Konstantin A. Chastagnol, Latomus 25, 1966, 537-552. Arcadius und Honorius als ubique vincentes: CIL VIII 5341 = ILS 5907 mit add.

${ }^{25}$ Siehe dazu G. Alföldy, in: W. Eck (Hrsg.), Religion und Gesellschaft in der römischen Kaiserzeit. Kolloquium zu Ehren von Friedrich Vittinghoff, Köln - Wien 1989, 60-63 = dens., Die Krise des Römischen Reiches. Geschichte, Geschichtsschreibung und Geschichtsbetrachtung. Ausgewählte Beiträge (Heidelberger Althistorische Beiträge und Epigraphische Studien 5), Stuttgart 1989, 354-356. ${ }^{26} \mathrm{CIL}$ VI 40416 (920 cf. $31203=$ ILS 216).

${ }^{27}$ G. Alföldy, Zeitschrift fưr Papyrologie und Epigraphik 109, 1995, 195-226 = in: R. Rea (Ed.), Rota Colisei. La valle del Colosseo attraverso i secoli, Milano 2002, 14-35; CLL VI 40454; vgl. jetzt L. H. Feldman, Biblical Archaeology Review 27, 4, 2001, 20-31 und 60-61; G. Alföldy, ebd. 27, 6, 2001, 16 f. ${ }^{28}$ Gellius, Noctes Atticae 13,25,1; vgl. G. Alföldy, Zeitschrift für Papyrologie und Epigraphik 109, 1995, 220 mit Literatur.

${ }^{29}$ Meilensteine des Augustus an der via Augusta und diejenigen seiner ersten Nachfolger, die den Ausbau dieser Straße vervollständigten, mit ähnlicher Beschriftung: CIL II $4701=$ ILS 102 und CLL II 4703 usw., siehe P. Sillières, in: R. Étienne (Ed.), Épigraphie hispanique. Problèmes de méthode et d'édition (Publications du Centre Pierre Paris 10), Paris 1984, 275 und bes. dens., Les voies de communication de l'Hispanie méridionale (Publications du Centre Pierre Paris 20), Paris 1990, 57 und im Katalog 68-118; zum Verlauf und zum Ausbau dieser Straße siehe ebd. 260-338. 580-589; vgl. jetzt A. U. Stylow - R. Atencia Páez - J. C. Vera, in: Akten des Kolloquiums „Siedlung und Verkehr“,
} 
Tiberius, Claudius, Domitian und Trajan in der Donauschlucht am Eisernen Tor an der zuerst als Holz- und Seilkonstruktion errichteten, unter Trajan in die Felswand gehauenen Straße, ${ }^{30}$ die Inschrift desselben Herrschers, die die Regulierung der Donau in der genannten Schlucht verewigt, ${ }^{31}$ oder an die Inschrift der Trajanssäule, wonach Trajan, um für sein forum Platz zu schaffen, einen Berg abtragen lie $\beta^{32}$

Als Botschaften der Macht erst recht aussagekräftig sind die Inschriften, die, schon seit den Lebzeiten des Augustus, vom Kult des numen Augusti, der göttlichen Macht des Herrschers, zeugen ${ }^{33}$ Gleiches gilt für die Inschriften, die auf den Kaiserbasen seit dem frühen 3. Jahrhundert die Bereitschaft der Untertanen bekunden, im Sinne der devotio ihr Leben für das numen des Kaisers zu opfern; ${ }^{34}$ das hat freilich, wenn auch in anderer Weise, nach dem Text des auch epigraphisch festgehaltenen Treueeides für den Kaiser von den Untertanen schon Augustus abverlangt. ${ }^{35}$ Die Inschrift aus Rom, die dort an Caracallas 25. Geburtstag, anläßlich seiner Rückkehr von dem siegreichen Feldzug in Britannien, von einem Verein kleiner Leute Deo Imp(eratori) Caes(ari) M(arco) Aurel/io Divi Septimi Severi f(ilio), OJptimo Antonino Pio, sideribu[s in terram delapso, T]onitratori Aug(usto), orbis terrarum [propagatori, domino] maximo mit dem Wunsch gesetzt wurde, daß er hundert Jahre lang glücklich regieren möge, zeigt, wie weit die

Festschrift Heinz E. Herzig, im Druck (A. U. Stylow danke herzlich ich für die Überlassung des noch ungedruckten Manuskriptes). Zur Benennung von Infrastrukturanlagen wie Straßen und Aquädukten nach Augustus vgl. G. Alföldy, Gymnasium 98, 1991, 301 und dens., Studi sull'epigrafia augustea e tiberiana (Anm. 21) 63 .

${ }^{30}$ J. Šašel, Opera Selecta (Situla 30), Ljubljana 1992, 259-276.

${ }^{31}$ J. Šašel, Journal of Roman Studies 63, 1973, 80-85 = ders., Opera Selecta (Anm. 30) 298-304.

${ }^{32} \mathrm{CIL}$ VI 960 cf. p. 4310 f. (mit Bibliographie) = ILS 294.

${ }^{33}$ Siehe dazu bes. D. Fishwick, The Imperial Cult in the Latin West. Studies in the Ruler Cult of the Western Provinces of the Roman Empire II 1, Leiden - New York - København - Köln 1991, 375422, außerdem dens., Britannia 25, 1994, 127-141; Uberblick über die fruhere Literatur bei W. Pötscher, in: Aufstieg und Niedergang der römischen Welt II 16, 1, Berlin - New York 1978, 380-387. Vgl. jetzt G. Alföldy - J. M. Abascal, in: J. M. Abascal - G. Alföldy (Eds.), El Arco romano de Medinaceli (Soria, Hispania citerior) (Bibliotheca Archaeologica Hispanica 18), Madrid 2002, 99-102.

${ }^{34}$ Zur einschlägigen Formel numini maiestatique eius devotissimus 0 . ä. siehe bes. H.-G. Gundel, Epigraphica 15, 1953 (1955), 128-150; weitere Literatur in CIL VI p. 4320 (zu CIL VI 1057-1058) und unter CIL VI 40621.

${ }^{35}$ P. Herrmann, Der römische Kaisereid. Untersuchungen zu seiner Herkunft und Entwicklung (Hypomnemata 20), Göttingen 1968. Vgl. M. Clauss, Kaiser und Gott. Herrscherkult im römischen Reich, Stuttgart 2001, 225-227. 
Anbetung des bereits zu Lebzeiten vergotteten Kaisers schon lange vor Aurelian gehen konnte. ${ }^{36}$

Zahlreiche Inschriften, die der Lobpreisung der Herrscher dienten, illustrierten Größe und Glanz der kaiserlichen Macht auch durch ihr äußeres Erscheinungsbild. Oft fällt schon die Größe der Buchstaben auf. Den „Rekord“ hält die Inschrift Agrippas, immerhin eines Kollegen des Augustus in der Ausübung der Macht, auf dem Pantheon, mit rund $70 \mathrm{~cm}$ hohen Buchstaben. Hier sei die Buchstabenhöhe einiger Bauinschriften von Kaisern und Mitgliedern des Kaiserhauses aus Rom und einigen Provinzen genannt. Bauinschrift des von Tiberius restaurierten Castor- und Pollux-Tempels: bis zu $53 \mathrm{~cm}$; Hadriansbogen bei Scythopolis in Judäa: bis zu $41 \mathrm{~cm}$; Titusbogen: bis zu $40 \mathrm{~cm}$; Augustusmonument in La Turbie: bis zu $37 \mathrm{~cm}$; Bogen von Medinaceli in Spanien: ungefähr $35 \mathrm{~cm}$; Aquädukt von Segovia: 32 bis $35 \mathrm{~cm}$; Inschrift des Tiberius vom forum Romanum: bis zu $34 \mathrm{~cm}$; Severusbogen in Rom: ungefähr $30 \mathrm{~cm}$; Claudiusbogen ebenda: ca. bis zu 30 $\mathrm{cm}$; Trajansinschrift auf der Tajo-Brücke bei Alcantará in Lusitanien: bis zu $30 \mathrm{~cm}$; Inschrift vom Nerva-forum: $28 / 30 \mathrm{~cm}$; Siegesmonument von Actium: $28 \mathrm{~cm}$; Inschrift des Augustus vom forum Romanum: bis zu 26,5 cm; MarsUltor-Tempel: $23 \mathrm{~cm} .{ }^{37}$ Die Buchstaben nichtkaiserlicher Inschriften waren, bis auf ganz wenige Ausnahmen, kleiner. Zugleich kennen wir einige Inschriften, in denen der Name eines Kaisers sozusagen nur en passant genannt, aber mit größeren Buchstaben als der übrige Text geschrieben wird; ähnlich sind in der erwähnten Inschrift Caracallas seine eigenen Worte durch größere Buchstaben hervorgehoben. ${ }^{38}$ Zahlreiche Inschriften der Kaiser beeindruckten den Betrachter auch dadurch, daß sie aus vergoldeten Bronzebuchstaben bestanden. Diese luxuriöse Technik diente vor allem der Verherrlichung der kaiserlichen Herrschaft. Nicht zufällig breitete sich der

${ }^{36}$ CIL VI 40638 (= 1080), siehe ausfuhrlich G. Alföldy, in: G. Bonamente - M. Mayer (Eds.), Historiae Augustae Colloquium Barcinonense MCMXCIII. Atti dei Convegni sulla Historia Augusta (Historiae Augustae Nova Series IV), Bari 1996, 9-36.

${ }^{37}$ Die angeführten Daten siehe bei Horster, a.a.O. (Anm. 3) 15 mit Anm. 39 bzw. bei W. Eck - G. Foerster, Journal of Roman Archaeology 12, 1999, 304; für die stadtrömischen Inschriften siehe die Angaben in den Addenda et corrigenda zu den bereits früher edierten bzw. unter den neu edierten Inschriften im Band CIL VI 8, 2; Segovia und Medinaceli: G. Alföldy, Die Bauinschriften des Aquäduktes von Segovia und des Amphitheaters von Tarraco (Madrider Forschungen 19, Berlin New York 1997), 22. 25.41 sowie Alföldy - Abascal, in: J. M. Abascal - G. Alföldy (Eds.), El Arco romano de Medinaceli (Anm. 33) 87.

${ }^{38}$ Siehe Anm. 36. Mit größeren Buchstaben wird z.B. der Name des Augustus in der Cursus honorum - Inschrift eines Senators seiner Zeit hervorgehoben: M. Buonocore, L' Abruzzo e il Molise in età romana. Tra storia ed epigrafia II (Deputazione Abruzzese di Storia Patria, Studi e Testi 21/2), L' Aquila 2002, 886-900 mit Fig. 60. 
Gebrauch der litterae aureae, wie diese Buchstaben hießen, seit dem Jahre $17 \mathrm{v}$. Chr. aus, als mit den ludi saeculares die neue aurea aetas eingeläutet wurde. Wie die Symbolik der aurea templa oder der aurea Roma, sollten auch die goldenen Buchstaben den Glanz des neuen Goldenen Zeitalters und seines allmächtigen Schöpfers vergegenwärtigen. ${ }^{39}$

Freilich wurden Macht und Erhabenheit der Herrscher nicht nur durch die Größe oder den goldenen Glanz der Buchstaben, sondern oft auch durch die besondere Dimension der Inschriften verkündet. So betrug die Länge der Inschriften des Aquäduktes von Segovia ungefähr $17 \mathrm{~m}$, des Mars Ultor-Tempels und des Castor- und Pollux-Tempels in Rom 21 bzw. $27 \mathrm{~m}$. Die Inschrift des Augustusmonuments von Actium war fast $60 \mathrm{~m}$ lang. Das alles wurde weit übertroffen durch die Podiumsinschrift des Amphitheaters von Tarraco, die die Nomenklatur und Rangtitulatur Elagabals enthielt, mit ihrer Länge von $147 \mathrm{~m} .{ }^{40}$ Es ist eine Ironie der Geschichte, daß dieses größte epigraphische Monument der Antike zugleich eines der kurzlebigsten war: Die im Jahre 221 angefertigte Inschrift wurde schon ein Jahr später, nach Elagabals Sturz, vernichtet; erhalten blieben nur die Namen der göttlichen Vorgänger des Kaisers und am Schluß der Hinweis auf die Erneuerung des Bauwerkes.

Die meisten bisher erwähnten Beispiele machen deutlich, daß die Inschriften ihre Botschaft erst recht deshalb verkünden konnten, weil sie, wie schon die Res Gestae Divi Augusti, oft zu außergewöhnlichen Monumenten gehörten, die ebenfalls die Größe der kaiserlichen Macht unterstrichen. Das gilt für die Kolossalstatuen, die in Rom als ein Privileg für die Herrscher galten; Beispiele sind etwa die Basen für monumentale Statuen Trajans auf

\footnotetext{
${ }^{39} \mathrm{Zu}$ Inschriften dieser Art siehe - jeweils mit weiterer Literatur - G. Alföldy, Der Obelisk auf dem Petersplatz, ein historisches Monument der Antike (Sitzungsberichte der Heidelberger Akademie der Wissenschaften, Philosophisch-historische Klasse, Jahrgang 1990, Bericht 2), Heidelberg 1990, bes. 21-27; dens., Gymnasium 98, 1991, 297-299; dens., Zeitschrift für Papyrologie und Epigraphik 94, 1992, 231-248; dens., ebd. 109, 1995, 195-226, bes. 205 f.; dens., Bauinschriften (Anm. 37) 1-51, bes. 4-8 und 26-31; dens., Biblical Archaeological Review 27, 6, 2001, 16 f.; J. M. Abascal - G. Alföldy R. Cebrián, Archivo Español de Arqueología 74, 2001, 117-130; Alföldy - Abascal, in: J. M. Abascal - G. Alföldy (Eds.), El Arco romano de Medinaceli (Anm. 33), bes. 80-98.

${ }^{40}$ Segovia: G. Alföldy, Bauinschriften (Anm. 37) 4. Inschriften des Mars Ultor- und des Dioskurentempels: Siehe bes. dens., Studi sull'epigrafia augustea e tiberiana (Anm. 21) 17-24 bzw. 39-58 und CIL VI 40311 bzw. 40339. Monument in Actium: W. M. Murray - Ph. M. Petsas, Octavian's Campsite Memorial for the Actian War (Transactions of the American Philosophical Society 79, 4), Philadephia 1989, 62 ff., bes. 74. Amphitheater von Tarraco: G. Alföldy, Bauinschriften 72. Weitere Angaben für die Länge kaiserlicher Bauinschriften bei Horster, a.a.O. (Anm. 3) 15-17.
} 
seinem forum. ${ }^{41}$ Auch große Tempel, Bögen, Siegesdenkmäler, Amphitheater, Aquädukte, Brücken, aber auch Grenzbauten wie z. B. die Hadriansmauer, einzelne Festungen oder sogar Wachtürme, an denen Inschriften mit Namen der Herrscher Roms zu lesen waren, konnten Respekt vor den Machthabern erwecken, wie etwa die Bauinschriften der aqua Claudia an der Porta Maggiore $^{42}$ oder des Kastells Aalen am rätischen Limes. ${ }^{43}$ Gleiches galt für so außergewöhnliche Inschriftenträger wie Säulen oder Obelisken. ${ }^{44}$ Als Beispiel sei hier der Vatikan-Obelisk genannt, ${ }^{45}$ der ursprünglich in Alexandria stand, wo ihn im Frühherbst $30 \mathrm{v}$. Chr. Cornelius Gallus, damals praefectus fabrum Oktavians, mit einer lateinischen Inschrift versehen und einweihen ließ (nach dem Tode des Augustus wurde diese Inschrift bekanntlich durch eine Widmung an Divus Augustus und seinen Nachfolger Tiberius ersetzt). Dieses unter Caligula nach Rom gebrachte Monument zeigt uns exemplarisch, wie Textinhalt, Beschriftungstechnik, Inschriftenträger und architektonischer Kontext zusammen der Verherrlichung eines Machthabers dienen konnten. Im kurzen Text war gleich zweimal der Name des Imperator Caesar Divi filius zu lesen, auf dessen Befehl (iussu) das forum Iulium, ein nach ihm benannter Repräsentationsplatz, errichtet wurde; die Inschrift bestand aus litterae aureae, die damals, erst recht in Ägypten, ein Novum darstellten; der Obelisk galt als Symbol für die Macht göttlicher Herrscher; wie der Montecitorio-Obelisk mit einem Globus und Dorn an der Spitze verkündete er die Weltherrschaft und dürfte auf dem forum Iulium Alexandrias, als Vorbild für das solarium Augusti in Rom, der Gnomon einer riesigen Sonnenuhr gewesen sein. Wie auch sonst oftmals, wurde die Aussagekraft der Inschrift hier durch die optische Wirkung des Monuments erhöht. Umgekehrt war das volle Verständnis der Botschaft eines Bauwerkes erst dank seiner Inschrift möglich. Als der in Ovids Fasti vom Himmel herabgestiegene Mars seinen von Augustus auf dessen forum gebauten Tempel besichtigt, entzücken ihn zuerst die Fassade, dann die

${ }^{41}$ CIL VI $959 a-c$ cf. p. 4310. Über monumentale Statuen und ihre Postamente bereitet B. Ruck (Heidelberg) eine Dissertation vor.

${ }^{42}$ CIL VI 1256-1258 cf. p. 4365 mit Bibliographie = ILS 218.

${ }^{43}$ Siehe dazu G. Alföldy, Fundberichte aus Baden-Württemberg 14, 1989, 293-338 (mit einem Beitrag von V. Habicht-Weinges) und dens., in: P. Barceló - V. Rosenberger (Hrsg.), Humanitas - Beiträge zur antiken Kulturgeschichte. Festschrift für Gunther Gottlieb zum 65. Geburtstag (Schriften der Philosophischen Fakultäten der Universität Augsburg 65), München 2001, 27-44. Zu ähnlichen Inschriften aus Limeskastellen vgl. M. Reuter, Saalburg Jahrbuch 48, 1995, 26-51.

${ }^{44}$ Säulen: Vgl. etwa die Inschriften der columna Traiani (siehe oben, Anm. 32) und der Säule des Antoninus Pius (CIL VI 1004 cf. p. 4315 mit Bibliographie). Obelisken in Rom: Siehe C. D'Onofrio, Gli obelischi di Roma, 2. ed., Roma 1967; vgl. J.-C. Grenier, in: E. M. Steinby (Ed.), Lexicon Topographicum urbis Romae III, Roma 1996, 355-359.

${ }^{45}$ G. Alföldy, Der Obelisk auf dem Petersplatz (Anm. 39) mit weiterer Literatur. 
Innenausstattung des Bauwerkes. Dann kommt er aus dem Tempel heraus und liest die Inschrift; als er dort den Namen des Bauherrn, Augustus, sieht, kommt ihm der Tempel noch größer vor. ${ }^{46}$

Die Res Gestae, die Obelisken mit ihrem Umfeld und selbst die Inschrift des Mars Ultor-Tempels im Kontext des forum Augustum exemplifizieren auch die Bedeutung des breiteren architektonischen und topographischen Rahmens, in dem eine sog. „Kaiserinschrift“ ihre oft einzigartige Wirkung entfalten konnte. Hierfür gibt es viele weitere Beispiele. In Tarraco standen die mit Widmungsinschriften versehenen Herrscherstatuen innerhalb des monumentalen Bezirkes für den Provinziallandtag der Hispania citerior auf der obersten Terrasse der Stadt, vor dem Augustustempel; sie beherrschten die Stadt zusammen mit diesem Tempel. ${ }^{47}$ Das Augustusmonument von La Turbie war in seiner imposanten Lage schon aus weiter Entfernung sichtbar. ${ }^{48}$ Der Aquädukt in Segovia, der m. E. eine Inschrift Trajans trug, führte vor Augen, wie die kaiserliche Macht Raum und Natur überwinden konnte. ${ }^{49}$

Genannt sei hier auch die bekannte Inschrift des Pontius Pilatus aus Caesarea in Israel, die uns den Schlüssel für das Verständnis der Herrschersymbolik im Hafen der genannten Stadt bietet. ${ }^{50}$ Ich hoffe gezeigt zu haben, daß die Pilatus-Inschrift die Bauinschrift eines Leuchtturmes im Hafen Caesareas war, der an der Spitze der Nordmole stand und nach Tiberius als Tiberieum benannt wurde, entsprechend dem großen Leuchtturm an der Spitze der Südmole mit dem Namen Druseum, benannt nach Drusus, dem Bruder des Tiberius. ${ }^{51}$ Die Symbolik kann erst im breiteren Kontext begriffen werden. Wie wir von Flavius Iosephus wissen, standen vor beiden

\footnotetext{
${ }^{46}$ Ovidius, Fasti 5,567 f. Zu der - wiederhergestellten - Inschrift siehe die Angaben oben in Anm. 40.

${ }^{47}$ Siehe hierzu G. Alföldy, jeweils mit Hinweis auf weitere Literatur zur Topographie, in: I. Rodà (Ed.), Tarraco, puerta de Roma. Exposición organizada por la Fundación "La Caixa", Tarragona, 13 de septiembre de 2001 - 6 de enero de 2002, Barcelona 2001, 30; dens., in: L. Hernández Guerra - L. Sagredo San Eustaquio - J. M. ${ }^{a}$ Solana Sáinz (Eds.), Actas del I Congreso Internacional de Historia Antigua "La Península Ibérica hace 2000 años" (Anm. 2) 71; vgl. dens., in: G. Brands et al. (Eds.), Rom und die Provinzen. Gedenkschrift für Hanns Gabelmann (Beihefte der Bonner Jahrbücher 53), Mainz 2001, $146 \mathrm{f}$.

${ }^{48} \mathrm{Zu}$ diesem Monument siehe N. Lamboglia, Le Trophée d'Auguste à La Turbie, 3. ed., Bordighera 1965; J. Formigé, Le trophée des Alpes (La Turbie) (Gallia Suppl. 2), Paris 1966; zur Inschrift vgl. Šašel, Opera Selecta (Anm. 30) 288-297.

${ }^{49}$ Alföldy, Bauinschriften (Anm. 37) 1-51; siehe auch dens., Zeitschrift für Papyrologie und Epigraphik 94, 1992, 231-248.

${ }^{50}$ G. Alföldy, Scripta Classica Israelica 18, 1999, 85-108 und ebd. 21, 2002, 133-148.

${ }^{51}$ Druseum: Iosephus, Bellum Iudaicum 1,412 und Antiquitates 15,336.
} 
Molensspitzen jeweils drei auf Säulen gestellte Statuen, ${ }^{52}$ die wohl Augustus und Livia einmal zusammen mit Tiberius, das andere Mal zusammen mit Drusus darstellten. Der Hafen hieß portus Augusti; die Stadt wurde nach Augustus Caesarea benannt; ihr prächtigstes Bauwerk, auf einer Erhöhung über dem Hafen, war das Augusteum, das die Seefahrer nach Iosephus bereits aus der Ferne sehen konnten. Die Botschaft der Gesamtanlage einschließlich der Pilatus-Inschrift ist unmißverständlich. Wie die nach Tiberius und Drusus benannten Türme den Eingang in den Augustushafen der Cäsarenstadt mit dem Tempel des Augustus schützen, so wachen Tiberius und Drusus - der eine zu Pilatus' Zeiten als Herrscher, der andere aus dem Jenseits - über das Erbe des Augustus. Tagsüber wurde diese Assoziation durch den Anblick des Tiberieum und des Druseum mit den ihnen vorgelagerten Kaiserstatuen, aber auch mit Hafen, Stadt und Tempel des Augustus dahinter vermittelt. Bei Nacht konnte man das Licht der nach Tiberius und Drusus benannten Leuchttürme als ein Symbol für das kosmische Licht empfinden, das diese beiden leuchtenden Sterne des Herrscherhauses ausstrahlten: Sie, nach der consolatio Liviae die concordia sidera, ${ }^{53}$ waren die Abbilder der wie sie auch nach dem Tod des jüngeren Bruders unzertrennbaren Brüder Castor und Pollux, deren Sternbilder den Seefahrern in der Nacht den Weg wiesen.

Ein eindrucksvolles Beispiel für die Herrschaftssymbolik ist auch der Bogen von Medinaceli $150 \mathrm{~km}$ nordöstlich von Madrid, über dem Tal, in dem die Straße Augusta Emerita - Caesaraugusta führte. ${ }^{54}$ Der Bogen, Tor einer römischen Stadt vielleicht mit dem Namen Ocilis, ist aus der Ferne sichtbar; durch seine drei Durchgänge öffnet sich ein Blick auf die endlos wirkende Hochebene Kastiliens. Die aus litterae aureae komponierte Inschrift, deren Wiederherstellung nach einer genauen Aufnahme ihrer Verdübelungsspuren vor kurzem gelungen sein dürfte, verherrlichte auf der Stadtseite das numen Augustum, die göttliche Macht der Herrscher Roms, auf der Talseite das numen des regierenden Kaisers. Die Macht der Kaiser über die Oikumene hätte kaum klarer zum Ausdruck gebracht werden können als durch dieses Zusammenspiel von Text, goldenen Buchstaben, Monument und Landschaft.

52 Iosephus, Bellum Iudaicum 1,413; zu den Fundamenten für diese Statuen siehe auch dens., Antiquitates 15,338.

${ }^{53}$ Consolatio ad Liviam (Epicedion Drusi) 283.

${ }^{54}$ J. M. Abascal - G. Alfoldy (Eds.), El Arco romano de Medinaceli (Anm. 33). 
Verstärkt wurde die Aussagekraft der „Kaiserinschriften“ auch durch ihre große Zahl. Nach der Zusammenstellung der einschlägigen Denkmäler durch J. M. Højte kennen wir aus dem Zeitraum von Augustus bis Commodus mehr als 2200 beschriftete Basen von Statuen der Augusti. ${ }^{55}$ Zusammen mit den späteren Kaiserbasen und den Postamenten für Statuen der Augustae und Caesares kämen wir auf etwa 5000 Stücke. Wenn jeder allgemein anerkannte Kaiser in jeder Stadt eine Statue mit Inschrift bekam, wären das viele Zehntausende von Widmungen. Vielerorts gab es freilich mehr als nur eine Statue furr einen Kaiser, so allein in Athen Hunderte von Hadriansstatuen. ${ }^{56}$ Die stereotypen Inschriften der Meilensteine wiederholten die Nomenklatur und Titulatur der Kaiser, wenn auch nicht unbedingt Meile für Meile, so doch sehr häufig, beispielsweise in Hispanien entlang der via Augusta von ihrem Beginn in den Pyrenäen bis zu ihrem Endpunkt in Gades. ${ }^{57}$ Von herausragenden Inschriften, die der Verherrlichung des Kaisershauses dienten, gab es auch Kopien an anderen Orten - nicht nur von den Res Gestae, sondern z. B. auch vom Ehrenschild des Augustus. ${ }^{58}$ Dazu kamen die Abschriften offizieller Dokumente wie der Senatsbeschlüsse zu Ehren des verstorbenen Germanicus, die in allen Kolonien und wohl auch in allen Municipien publik gemacht wurden, ${ }^{59}$ oder das Senatus consultum de Cn. Pisone patre, mehr eine Laudatio auf das Herrscherhaus als ein Gerichtsurteil, das in allen Städten der Baetica angeschlagen war. ${ }^{60}$

\footnotetext{
ss Siehe oben Anm. 3. Zur großen Zahl der Kaiserstatuen, die auf entsprechend beschrifteten Postamenten standen, vgl. auch Th. Pekáry, Das römische Kaiserbildnis in Staat, Kult und Gesellschaft, dargestellt anhand der Schriftquellen (Das römische Herrscherbild III), Berlin 1985, bes. 4-12. 22-28. 42-65.

${ }^{56}$ Vgl. Pekáry, a.a.O. (Anm. 55) 7. 27. 35.44 usw.

${ }^{57} \mathrm{Zu}$ den Meilensteinen an dem in der Hispania citerior befindlichen Abschnitt dieser Straße siehe die Materialsammlung bei F. Pallí Aguilera, La Vía Augusta en Cataluña, Bellaterra 1985 sowie J. Lostal Pros, Los miliarios de la provincia Tarraconense (conventos Tarraconense, Cesaraugustano, Cluniense y Cartaginense), Zaragoza 1992. Meilensteine der via Augusta in der Baetica: Siehe Sillières, Les voies de communication de l'Hispanie méridionale (Ann. 29) 68-118 Nr. 1-61.

${ }^{58}$ Vgl. G. Alföldy, Gymnasium 98, 1991, 308 mit Anm. 49; dazu jetzt auch CIL VI 40365 (dort auch Näheres zu anderen Kopien) und 40366, ausführlich dazu Panciera, in: H. von Hesberg - S. Panciera, Das Mausoleum des Augustus (Anm. 14) 113-118 Nr. 9 und 171 f. Nr. LIV. Siehe noch Kienast, Augustus (Anm. 4) 96, u. a. mit Hinweis auf eine Kopie auch in Ostia.

${ }^{59}$ CIL VI 911 = 31199 mit weiterer Literatur; zu den Kopien siehe AE 1949, 215 = 1952, 164 (Tabula Hebana) und AE 1984, 508 = CILA II 927 (Tabula Siarensis), dazu ausführlich Á. Sánchez-Ostiz Gutierrez, Tabula Siarensis. Edición, traducción y comentario (Colección Mundo Antiguo, N. S. 4), Pamplona 1999; vgl. noch bes. J. González - J. Arce (Eds.), Estudios sobre la Tabula Siarensis, Madrid 1988.

${ }^{60} \mathrm{CIL} \mathrm{II} / 5$, 900; siehe ausführlich Eck - Caballos - Fernández, Das senatus consultum de $\mathrm{Cn}$. Pisone patre (Anm. 17), zur Publikation der Kopien in der Baetica: 279-287, ebenso A. Caballos - W. Eck F. Fernández, El senadoconsulto de Gneo Pisón padre, Sevilla 1996, zur Publikation der Kopien in der
} 
Man kann hinzufügen: Ruhm und Macht der Kaiser Roms wurden hin und wieder sogar außerhalb des Reiches durch Inschriften verkündet. Erinnert sei hier an die Siegesinschrift im Felsen von Trenčin in der Slowakei, in Laugaricio im Quadenland, $120 \mathrm{~km}$ nördlich von der Donaugrenze, mit der Widmung Victoriae Augustoru(m), dediziert im Winter 179/180 von einer Legionsabteilung, die vor dem für das nächste Frühjahr geplanten Feldzug gegen die Germanen dort überwintert hat. ${ }^{61}$ Bei solchen Dokumenten, so z. B. auch bei den im Kaukasus gesetzten Inschriften mit der Nennung der Kaiser Domitian, Mark Aurel oder Commodus, ${ }^{62}$ könnte man sich fragen, wer diese Texte überhaupt lesen konnte. Das trifft aber z. B. auch für den von Cornelius Gallus unmittelbar nach der Eroberung Ägyptens in Alexandria dedizierten Obelisken mit seiner lateinischen Inschrift zu, zumal Gallus sein eigenes Siegesdenkmal in Philae einige Jahre später gleichzeitig in lateinischer, griechischer und ägyptischer Sprache beschriften ließ, damit den Text dort jedermann verstehen konnte. ${ }^{63}$ Die Frage nach der Rezeption der Inschriften stellt sich freilich, angesichts des weit verbreiteten Analphabetismus, für das ganze Imperium. ${ }^{64}$ Was die Reichsbevölkerung betriff, können wir wohl davon ausgehen, daß viele Menschen, die die lateinischen - oder im Osten die griechischen - Texte nicht lesen konnten, zumindest die wichtigsten Begriffe wie die überaus häufig vorkommende, auch durch die Münzen bekannt gewordene Nomenklatur bzw. Titulatur Imp. Caesar Augustus (oder Autokrator Kaisar Sebastos) dennoch

Baetica: 133-141; zu den Kopien in der Baetica siehe auch A. U. Stylow - S. Corso Pérez, Chiron 29, 1999, 23-28.

${ }^{61}$ Siehe CIL III 13439 = J. Č ̌ska - R. Hošek, Inscriptiones Pannoniae superioris in Slovacia Transdanubiana asservatae (Opera Universitatis Purknynianae Brunensis, Facultas Philosophica 125), Brno 1967, Nr. 2; grundlegend dazu H.-G. Pflaum, Les carrières procuratoriennes équestres sous le Haut-Empire romain I, Paris 1960, 476-494 Nr. 181 bis, bes. 493.

${ }^{62}$ AE 1951, 263; AE 1910, 161 = ILS 9117; CIL III 6052 = ILS 394; zu der an erster Stelle genannten Inschrift siehe bes. B. Isaac, The Limits of Empire. The Roman Army in the East, 2. ed., Oxford 1992, $44 \mathrm{f}$. und F. Bertrandy - B. Rémy, in: Y. Le Bohec - C. Wolff (Eds.), Les légions de Rome sous le Haut-Empire. Actes du Congrès de Lyon (17-19 septembre 1998), Lyon 2000, 255; zu den beiden anderen Inschriften E. L. Wheeler, ebd. 300.

${ }^{63}$ CIL III 14147,5 = IGRR I/II $1293=$ OGIS II $654=$ ILS 8995 = É. Bernand, Les inscriptions grecques et latines de Philae II. Haut et Bas Empire, Paris 1969, Nr. 128; vgl. Alföldy, Der Obelisk auf dem Petersplatz (Anm. 39) 96-100.

${ }^{64} \mathrm{Zu}$ der - in der Forschung umstrittenen - Dimension der Lesefähigkeit bzw. des Analphabetismus in der römischen Welt siehe bes. W. V. Harris, Ancient Literacy, Cambridge, Mass. - London 1989, bes. 175-322; zur Problematik des Zusammenhanges zwischen der Verbreitung der epigraphischen Denkmäler und der Schriftlichkeit ebd. 265-273. $287 \mathrm{f}$. 
erkennen konnten. ${ }^{65}$ Außerdem konnte man die Kaisernamen in einigen Teilen des Reiches auch in der eigenen Sprache und Schrift einzelner Bevölkerungsgruppen, beispielsweise punisch oder in Hieroglyphenschrift, wiedergeben ${ }^{66}$ Der Gebrauch des Lateins fur die Inschriften in soeben unterworfenen oder vorübergehend besetzten Gebieten erklärt sich dagegen wohl eher dadurch, daß so die Macht Roms zur Schau gestellt werden sollte: Der lokalen Bevölkerung muß zumindest soviel bekannt gewesen sein, daß die ihnen unverständlichen Schiftzeichen von der Präsenz der Römer und somit von der Macht Roms und seines Kaisers zeugen. ${ }^{67}$

Ein Kaiser wurde jedenfalls durch unvergleichlich mehr Inschriften gerühmt als selbst ein hochangesehener Senator oder Ritter. Dazu kommt freilich noch zusätzlich, daß die Herrscher auch in vielen Inschriften der Untertanen als diejenigen verherrlicht wurden, denen diese ihren sozialen Aufstieg einschließlich Ämter und Privilegien zu verdanken hatten. ${ }^{68}$ Zugleich nutzten die Herrscher dieses Medium für ihre eigenen Ziele in einer vielfach exklusiven Weise. Das zeigt sich nicht nur durch den Inhalt, die Größe, die Pracht oder die Plazierung ihrer epigraphischen Monumente, auch nicht nur durch ihre große Anzahl. In Rom gab es seit Augustus bis auf furr einige Nutzbauten keine Bauinschrift mehr, die andere Bauherren als die Mitglieder des Kaiserhauses verherrlicht hätten. ${ }^{69}$ Seit der mittleren

\footnotetext{
${ }^{65} \mathrm{Zu}$ der Möglichkeit, daß ganz einfache epigraphische Texte oder stereotype Wendungen selbst von Analphabeten erkannt und identifiziert werden konnten, vgl. W. V. Harris, Zeitschrift für Papyrologie und Epigraphik 52, 1983, 107-111. Uber eine „alphabétisation pauvre, largement répandu“" der Rezipienten von Inschriften siehe M. Corbier, in: L'Urbs. Espace urbaine et histoire ( ${ }^{\mathrm{er}}$ siècle av. J.-C. - III ${ }^{e}$ siècle ap. J.-C.). Actes du colloque international organisé par le Centre National de la Recherche Cientifique et l'École Française de Rome (Rome, 8-12 mai 1985), Paris - Roma 1987, 59 f.

${ }^{66} \mathrm{Zu}$ Bildern römischer Kaiser nach ägyptischer Auffassung - zu denen oft einschlägige Texte in ägyptischer Sprache und Schrift gehörten - siehe M. Alföldi, a.a.O. (Anm. 3) 166-170 mit weiterer Literatur. Bilingue Inschriften zu Ehren des Augustus in lateinischer und punischer Schrift in Lepcis Magna: IRT 321-323.

${ }^{67}$ Es sei gestattet, auf eine moderne Analogie hinzuweisen. Die - nicht selten aus vergoldeten Bronzebuchstaben bestehenden - Inschriften, die nach 1945 auf den sowjetischen Sieges- und Gedenkmonumenten der osteuropäischen Länder mit zyrillischen Buchstaben in russischer Sprache angebracht wurden, konnten zwar dort die meisten Menschen nicht lesen; ein Blick auf diese Monumente genügte jedoch, um sich die Herrschaftsvorstellung zu vergegenwärtigen, die diese Denkmäler und speziell auch ihre Beschriftung verkündeten.

${ }^{68}$ Siehe beispielsweise die zahlreichen Inschriften, in denen Offiziere und Soldaten des römischen Heeres die Kaiser als ihre persönlichen Wohltäter rühmen; dazu G. Alföldy, in: A. Chaniotis - P. Ducrey (Eds.), Army and Power in the Ancient World (Heidelberger Althistorische Beiträge und Epigraphische Studien 37), Stuttgart 2002, 123-150.

${ }^{69}$ W. Eck, in: F. Millar - E. Segal, Caesar Augustus. Seven Aspects, Oxford 1984, 129-167, bes. 137142 = ders., Tra epigrafia, prosopografia e archeologia. Scritti scelti, rielaborati ed aggiornati (Vetera 10), Roma 1996, 271-298, bes. 276-278.
} 
Regierungszeit des Augustus bekam dort auf den öffentlichen Plätzen und in den öffentlichen Bauten niemand mehr eine Ehrenstatue und somit auch keine Ehreninschrift außer den Vertrauten und Freunden der Herrscher, die die Errichtung derartiger Monumente für ihre führenden Anhänger - oft erst nach deren Tode - erlaubten. ${ }^{70}$ Auch gab es nach Augustus keinen Meilenstein mehr, der eine andere Person als den Kaiser als Bauherrn einer via publica verewigt hätte. ${ }^{71}$ Die Liste der kaiserlichen Vorrechte in der Nutzung des Mediums „Inschrift“ ließe sich fortsetzen.

Alles in allem eigneten sich die inschriftlichen Monumente der römischen Welt bestens dazu, die Macht der Herrscher zu vergegenwärtigen. Die Kaiser nutzten für die Festigung ihrer Herrschaft nicht nur die Macht der Bilder, sondern, in engster Verbindung damit, auch die Macht der Worte, die nicht nur gesprochen, sondern in bleibender Form festgehalten wurden, um ebenso wie die Bilder für die Ewigkeit Geltung zu haben. Somit übten die inschriftlichen Monumente, die zur Verherrlichung der Kaiser und ihrer Herrschaft eingesetzt waren, ebenso wie die gesamte epigraphische Kultur der Römer, die ihre Wertvorstellungen und Verhaltensnormen für künftige Generationen dokumentierte, eine wahrhaftig nicht zu unterschätzende systemstabilisierende Funktion aus. Man könnte sagen: Wie etwa heute der Prozeß der Vereinigung Europas zu einem nicht unbedeutenden Teil ein Werk der Medien ist, die diesen Prozeß Tag für Tag weiter verfolgen und seine Bedeutung in die Köpfe und Herzen der Menschen einhämmern, so festigten sich, mutatis mutandis, auch die kaiserliche Macht in Rom und damit das Imperium Romanum dank dem Einfluß der Medien der damaligen Zeit. Der epigraphischen Kultur Roms fiel dabei eine ähnlich entscheidende Rolle zu wie den Monumenten, zu denen die Inschriften gehörten.

Heidelberg, Dezember 2002

${ }^{70}$ G. Alföldy, in: G. Alföldy - S. Panciera (Eds.), Inschriftliche Denkmäler als Medien der Selbstdarstellung (Anm. 1) 11-46.

${ }^{71}$ G. Alföldy, Gymnasium 98, 1991, 300 f. 